\title{
A Deterministic Algorithm for Bridging Anaphora Resolution
}

\author{
Yufang Hou \\ IBM Research Ireland \\ yhoulie.ibm.com
}

\begin{abstract}
Previous work on bridging anaphora resolution (Poesio et al., 2004; Hou et al., 2013b) use syntactic preposition patterns to calculate word relatedness. However, such patterns only consider NPs' head nouns and hence do not fully capture the semantics of NPs. Recently, Hou (2018) created word embeddings (embeddings_PP) to capture associative similarity (i.e., relatedness) between nouns by exploring the syntactic structure of noun phrases. But embeddings_PP only contains word representations for nouns. In this paper, we create new word vectors by combining embeddings_PP with GloVe. This new word embeddings (embeddings_bridging) are a more general lexical knowledge resource for bridging and allow us to represent the meaning of an NP beyond its head easily. We therefore develop a deterministic approach for bridging anaphora resolution, which represents the semantics of an NP based on its head noun and modifications. We show that this simple approach achieves the competitive results compared to the best system in Hou et al. (2013b) which explores Markov Logic Networks to model the problem. Additionally, we further improve the results for bridging anaphora resolution reported in Hou (2018) by combining our simple deterministic approach with Hou et al. (2013b)'s best system $M L N I I$.
\end{abstract}

\section{Introduction}

Anaphora plays a major role in discourse comprehension and accounts for the coherence of a text. In contrast to identity anaphora which indicates that a noun phrase refers back to the same entity introduced by previous descriptions in the discourse, bridging anaphora or associative anaphora links anaphors and antecedents via lexico-semantic, frame or encyclopedic relations. Bridging resolution is the task to recognize bridging anaphors (e.g., distribution arrangements in
Example $1^{1}$ ) and find links to their antecedents (e.g., dialysis products in Example 1).

(1) While the discussions between Delmed and National Medical Care have been discontinued, Delmed will continue to supply dialysis products through National Medical after their exclusive agreement ends in March 1990, Delmed said. In addition, Delmed is exploring distribution arrangements with Fresenius USA, Delmed said.

Most previous empirical research on bridging (Poesio and Vieira, 1998; Poesio et al., 2004; Markert et al., 2003; Lassalle and Denis, 2011; Hou et al., 2013b) focus on bridging anaphora resolution, a subtask of bridging resolution that aims to choose the antecedents for bridging anaphors. For this substask, most previous work (Poesio et al., 2004; Lassalle and Denis, 2011; Hou et al., 2013b) calculate semantic relatedness between an anaphor and its antecedent based on word co-occurrence counts using certain syntactic patterns. However, such patterns only consider head noun knowledge and hence are not sufficient for bridging relations which require the semantics of modification. In Example 1, in order to find the antecedent (dialysis products) for the bridging anaphor "distribution arrangements", we have to understand the semantics of the modification "distribution".

Over the past few years, word embeddings gained a lot popularity in the NLP community. State-of-the-art word vectors such as word2vec skip-gram (Mikolov et al., 2013) and GloVe (Pennington et al., 2014) have been shown to perform well across a variety of NLP tasks, including textual entailment (Rocktäschel et al., 2016), reading

\footnotetext{
${ }^{1}$ All examples, if not specified otherwise, are from ISNotes (Markert et al., 2012). Bridging anaphors are typed in boldface, antecedents in italics throughout this paper.
} 
comprehension (Chen et al., 2016) and coreference resolution (Lee et al., 2017).

Recently, Hou (2018) found that these vanilla word embeddings capture both "genuine" similarity and relatedness, and hence they are not suitable for bridging anaphora resolution which requires lexical association knowledge instead of semantic similarity information between synonyms or hypernyms. Hou (2018) created word embeddings for bridging (embeddings_PP) by exploring the syntactic structure of noun phrases (NPs) to derive contexts for nouns in the GloVe model.

However, embeddings_PP only contains the word representations for nouns. In this paper, we improve embeddings_PP by combining it with GloVe. The resulting word embeddings (embeddings_bridging) are a more general lexical knowledge resource for bridging anaphora resolution. Compared to embeddings_PP, the coverage of lexicon in embeddings_bridging is much larger. Also the word representations for nouns without the suffix "PP" are more accurate because they are trained on many more instances in the vanilla GloVe. Based on this general vector space, we develop a deterministic algorithm to select antecedents for bridging anaphors. Our approach combines the semantics of an NP's head with the semantics of its modifications by vector average using embeddings_bridging. We show that this simple, efficient method achieves the competitive results on ISNotes for the task of bridging anaphora resolution compared to the best system in Hou et al. (2013b) which explores Markov Logic Networks to model the problem.

The main contributions of our work are: (1) a general word representation resource ${ }^{2}$ for bridging; and (2) a simple yet competitive deterministic approach for bridging anaphora resolution which models the meaning of an NP based on its head noun and modifications.

\section{Related Work}

Lexical/world knowledge for bridging: Hou et al. (2013a) explored various lexico-semantic features for bridging anaphora recognition. Hou (2016) proposed an attention-based LSTM model with pre-trained word embeddings for information status classification and reported moderate results for bridging recognition. Previous work on bridg-

\footnotetext{
${ }^{2}$ embeddings_bridging can be downloaded from https : //doi.org/10.5281/zenodo.1403164
}

ing anaphora resolution (Poesio et al., 2004; Lassalle and Denis, 2011; Hou et al., 2013b) explored word co-occurrence counts in certain syntactic preposition patterns to calculate word relatedness. For instance, the big hit counts of the query "the door of the house" in large corpora could indicate that door and house stand in a part-of relation. These patterns encode associative relations between nouns which cover a variety of bridging relations. Unlike previous work which only consider a small number of prepositions per anaphor, the PP context model (Hou, 2018) uses all prepositions for all nouns in big corpora. It also includes the possessive structure of NPs. In this paper, we further improve Hou (2018)'s embeddings_PP by combining it with the vanilla GloVe. The resulting word embeddings (embeddings_bridging) are a more general lexical knowledge resource for bridging resolution. In addition, it enables efficient computation of word association strength through low-dimensional matrix operations.

Bridging anaphora resolution: regarding the methods to select antecedents for bridging anaphors, Poesio et al. (2004) applied a pairwise model combining lexical semantic features as well as salience features to perform mereological bridging resolution in the GNOME corpus $^{3}$. To address the data sparseness problem (e.g., some part-of relations are not covered by WordNet), they used the Web to estimate the part-of relations expressed by certain syntactic constructions. Based on the method proposed by Poesio et al. (2004), Lassalle and Denis (2011) developed a system that resolves mereological bridging anaphors in French. The system was enriched with meronymic information extracted from raw texts. Such information was extracted in a bootstrapping fashion by iteratively collecting meronymic pairs and the corresponding syntactic patterns. Lassalle and Denis (2011) evaluated their system on mereological bridging anaphors annotated in the DEDE corpus and reported an accuracy of $23 \%$.

Markert et al. (2012) released a corpus called ISNotes which contains unrestricted bridging annotations. Based on this corpus, Hou et al. (2013b) proposed a joint inference framework for bridging anaphora resolution using Markov logic networks (Domingos and Lowd, 2009). The framework resolves all bridging anaphors in one document together by modeling that semantically re-

\footnotetext{
${ }^{3}$ The GNOME corpus is not publicly available.
} 


\begin{tabular}{l|l}
\hline Noun Phrases & Extracted Noun Pairs \\
\hline travelers in the train station & travelers_PP-station \\
travelers from the airport & travelers_PP-airport \\
hotels for travelers & hotels_PP-travelers \\
the destination for travelers & destination_PP-travelers \\
the company's new appointed chairman & chairman_PP-company \\
\hline
\end{tabular}

Table 1: Examples of noun phrases as well as the extracted noun pairs in embeddings_PP. Bold indicates the head noun of an NP.

lated anaphors are likely to share the same antecedent.

ISNotes is a challenging corpus for bridging. First, bridging anaphors are not limited to definite NPs as in previous work (Poesio et al., 1997, 2004; Lassalle and Denis, 2011). Also in ISNotes, the semantic relations between anaphor and antecedent are not restricted to meronymic relations. We therefore choose ISNotes to evaluate our algorithm for bridging anaphora resolution. Our approach is deterministic and simple, but achieves the competitive results compared to the advanced machine learning-based approach (Hou et al., 2013b). We also improve the result reported in Hou (2018) on the same corpus by combining our deterministic approach with the best system from Hou et al. (2013b).

Just recently, two new corpora (Rösiger, 2018a; Poesio et al., 2018) with bridging annotations have become available and we notice that the definitions of bridging in these corpora are different from the bridging definition in ISNotes. We apply our algorithm with small adaptations to select antecedents for bridging anaphors on these corpora. The moderate results demonstrate that $\mathrm{em}$ beddings_bridging is a general word representation resource for bridging.

\section{Word Representations for Bridging}

\subsection{Word Embeddings Based on PP Contexts (embeddings_PP)}

We briefly describe Hou (2018)'s embeddings_PP in this section. embeddings_PP released by $\mathrm{Hou}$ (2018) contains 100-dimensional vectors for 276k nouns. It is trained over 197 million noun pairs extracted from the automatically parsed Gigaword corpus (Parker et al., 2011; Napoles et al., 2012). The author generates these noun pairs by exploring the syntactic prepositional and possessive structures of noun phrases. These two structures encode a variety of bridging relations between anaphors and their antecedents. For instance, the prepositional structure in "the door of the house" indicates the part-of relation between "door" and "house". More specifically, for NPs containing the prepositional structure (e.g., $\mathbf{X}$ preposition $\mathbf{Y}$ ) or the possessive structure (e.g., $\mathbf{Y}$ 's $\mathbf{X}$ ), the author extracts the noun pair "X_PP-Y". Note that the head of the NP is always on the left and the noun modifier is always on the right. In addition, the suffix "PP" is added for the nouns on the left. Table 1 shows a few examples of noun phrases together with the extracted noun pairs.

Hou (2018) showed that the suffix "_PP" plays an important role for the model to learn the asymmetric relations between the head nouns and their noun modifiers from the extracted noun pairs. For instance, among the top five nearest neighbors in embeddings_PP, "president_PP" is mostly related to countries or organizations (e.g., "federation", "republic", or "USA"), while "president" is mostly related to words which have the same semantic type as "president" (e.g., "minister", "mayor", or "governor").

\subsection{Word Representations for Bridging (embeddings_bridging)}

embeddings_PP described in the previous section only contains word representations for nouns. To improve the coverage of lexical information, we create a general word representation resource embeddings_bridging by merging embeddings_PP with the original GloVe vectors trained on Gigaword and Wikipedia datasets. Specifically, given the 100 dimension word embeddings $\mathrm{em}$ beddings_PP and GloVe, we first create a 100 dimension vector $v_{\text {filler }}$ with the value of each dimension as $0.1^{4}$. Let $v 1_{w}$ represent the vector for the word $w$ in GloVe, $v 2_{w}$ represent the vector for the word $w$ in embeddings_PP, if a word $w$ appears

\footnotetext{
${ }^{4}$ Theoretically, any 100 dimension random vector with uniform distribution could be used as $v_{\text {filler }}$.
} 


\begin{tabular}{l|c|c}
\hline & embeddings_bridging & embeddings_PP \\
\hline dimension size & 200 & 100 \\
vocabulary size & 532,768 & 276,326 \\
word type & all & nouns \\
\hline
\end{tabular}

Table 2: Comparison between embeddings_bridging and embeddings_PP

\begin{tabular}{|c|c|c|c|c|c|}
\hline Category & Relation & $\begin{array}{l}\text { prototypical word } \\
\text { pair example }\end{array}$ & $\begin{array}{l}\text { embeddings } \\
\text { bridging }\end{array}$ & $\begin{array}{c}\text { embeddings } \\
\text { PP }\end{array}$ & GloVe \\
\hline$\overline{P A R T-W H O L E}$ & Object: Component & $\{$ face: nose $\}$ & 0.43 & 0.27 & 0.40 \\
\hline PART-WHOLE & Event: Feature & $\{$ wedding: bride $\}$ & 0.46 & 0.29 & 0.15 \\
\hline PART-WHOLE & Creature: Possession & \{author: copyright $\}$ & 0.23 & 0.14 & 0.21 \\
\hline PART-WHOLE & Activity: Stage & $\{$ buying: shopping $\}$ & 0.32 & 0.30 & 0.25 \\
\hline
\end{tabular}

Table 3: Spearman's rank correlation coefficient $\rho$ for typical PART-WHOLE bridging relations using embeddings_bridging, embeddings_PP and GloVe.

both in GloVe and in embeddings_PP, its vector in embeddings_bridging is the concatenation of $v 1_{w}$ and $v 2_{w}$. For the word $w_{1}$ which only appears in GloVe, its vector in embeddings_bridging is the concatenation of $v 1_{w_{1}}$ and $v_{\text {filler }}$. Finally, for the word $w_{2}$ which only appears in embeddings_PP (all the words with the suffix " $P P$ "), we construct its vector by concatenating $v_{\text {filler }}$ and $v 2_{w_{2}}$. The resulting 200 dimension word embeddings (embeddings_bridging) is a general lexical resource for bridging. Table 2 compares the main features between embeddings_bridging and embeddings_PP. In the next section, we will compare embeddings_bridging with embeddings_PP and the original GloVe on a few typical bridging relations in the task of measuring relational similarity (Jurgens et al., 2012). Moreover, in Section 5.3 and Section 5.4, we show that using embeddings_bridging yields better results than using $\mathrm{em}$ beddings_PP for bridging anaphora resolution.

\subsection{Measuring Relational Similarity on Typical Bridging Relations}

We evaluate our embeddings_bridging quantitatively using a few typical bridging relations from SemEval-2012 Task 2 (Jurgens et al., 2012). The shared task aims to rank word pairs by the degree to which they are prototypical members of a given relation class. For instance, given the prototypical word pairs \{wedding-bride, rodeo-cowboy, banquet-food $\}$ for the relation Event:Feature, we would like to know among the input word pairs $\{$ school-students, circus-clown, meal-food, lion$z o o\}$, which one represents the relation best.
SemEval-2012 Task 2 contains 79 relation classes chosen from Bejar et al. (1991). These relations fall into ten main categories, including SIMILAR, PART-WHOLE, CONTRAST and more. Each relation class is paired with a few prototypical word pairs and a list of around 40 word pairs which are ranked by humans according to their degree of similarity to the corresponding relation. We choose all typical bridging relations under the PART-WHOLE category and evaluate our embeddings_bridging in terms of ranking the list of word pairs for each relation. Spearman's rank correlation coefficient $\rho$ is used to evaluate a system by comparing the system's ranking of the word pairs against the gold standard ranking.

Following Zhila et al. (2013), we calculate the relational similarity between word pairs using cosine similarity. Let $\left(w_{1}, w_{2}\right)$ and $\left(w_{3}, w_{4}\right)$ be the two word pairs, $v_{1}, v_{2}, v_{3}, v_{4}$ be the corresponding vectors for these words. We first normalize all word vectors to unit vectors, then the relational similarity between $\left(w_{1}, w_{2}\right)$ and $\left(w_{3}, w_{4}\right)$ is calculated as:

$$
\frac{(v 1-v 2) \cdot(v 3-v 4)}{\|v 1-v 2\|\|v 3-v 4\|}
$$

For each chosen relation class, we rank the list of word pairs according to their mean relational similarity to the given prototypical word pairs. Table 3 shows the results of Spearman's rank correlation coefficient $\rho$ for each typical bridging relation using embeddings_bridging, embeddings_PP, and $G l o V e$, respectively. Note that when using embeddings_bridging and embeddings_PP, we add the suffix "PP" to the potential bridging anaphor for 


\begin{tabular}{l|l|l|l|l}
\hline \multicolumn{5}{c}{ bridging anaphor: distribution arrangements } \\
\hline Ante. Candidates & Head & Head + Modifiers & dist $_{h}$ & dist $_{h m}$ \\
\hline $\begin{array}{l}\text { the discussions between Delmed } \\
\text { and National Medical Care }\end{array}$ & $\{$ discussions $\}$ & $\{$ discussions $\}$ & 0.05 & -0.10 \\
\hline Delmed & $\{$ delmed $\}$ & $\{$ delmed $\}$ & - & - \\
\hline National Medical Care & $\{$ care $\}$ & $\{$ care $\}$ & $\mathbf{0 . 0 8}$ & 0.10 \\
\hline dialysis products & $\{$ products $\}$ & $\{$ dialysis, products $\}$ & 0.06 & $\mathbf{0 . 1 7}$ \\
\hline National Medical & $\{$ medical $\}$ & $\{$ medical $\}$ & 0.02 & -0.01 \\
\hline their & $\{$ their $\}$ & $\{$ their $\}$ & -0.05 & -0.01 \\
\hline their exclusive agreement & $\{$ agreement $\}$ & $\{$ exclusive, agreement $\}$ & 0.07 & 0.03 \\
\hline
\end{tabular}

Table 4: The cosine similarities between the bridging anaphor distribution arrangements and its antecedent candidates for Example 1. dist ${ }_{h}$ indicates the cosine similarity between $\{$ arrangements_PP $\}$ and the candidate head, dist $_{h m}$ the cosine similarity between $\{$ distribution_PP, arrangements_PP $\}$ and Head+Modifiers. "-_" means Delmed is not present in embeddings_bridging and therefore we neglect this candidate.

each word pair (e.g., \{wedding: bride_PP $\}$ ). As shown in Table 3, using embeddings_bridging performs better than both using embeddings_PP and using the vanilla GloVe vectors on these four partof relation classes. This partially indicates that embeddings_bridging could capture lexical knowledge for bridging relations.

\section{A Deterministic Algorithm for Bridging Anaphora Resolution}

In this section, we describe our deterministic algorithm based on embeddings_bridging for bridging anaphora resolution. For each anaphor $a$, we construct the list of antecedent candidates $E_{a}$ using NPs preceding $a$ from the same sentence as well as from the previous two sentences. Hou et al. (2013b) found that globally salient entities are likely to be the antecedents of all anaphors in a text. We approximate this by adding NPs from the first sentence of the text to $E_{a}$. This is motivated by the fact that ISNotes is a newswire corpus and globally salient entities are often introduced in the beginning of an article. We exclude an NP from $E_{a}$ if it is a bridging anaphor because a bridging anaphor is rarely to be an antecedent for another bridging anaphor. We also exclude NPs whose semantic types are "time" from $E_{a}$ if $a$ is not a time expression. This is because time expressions are related to a lot of words in the corpus in which we learned embeddings_bridging from. Therefore we only keep them as the antecedent candidates for bridging anaphors whose semantic types are "time" (see Example 2).

(2) As a presidential candidate in 1980 , George Bush forthrightly expressed his position on abor- tion in an interview with Rolling Stone magazine published that March.

Given an anaphor $a$ and its antecedent candidate list $E_{a}$, we predict the most semantically related NP among all NPs in $E_{a}$ as the antecedent for $a$. In case of a tie, the closest one is chosen to be the predicted antecedent.

The relatedness is measured via cosine similarity between the vector representation of the anaphor and the vector representation of the candidate. More specifically, given a noun phrase $n p_{1}$, we first construct a list $N$ which consists of the head and all common nouns (e.g., earthquake victims), adjectives (e.g., economical sanctions), and ed/ing participles (e.g., the collapsed roadway and the landing site) appearing before the head. If $n p_{1}$ contains a post-modifier NP $n p_{2}$ via the preposition "of", we also add the above premodifiers and the head of $n p_{2}$ to the list $N$ (e.g., the policies of racial segregation). Finally, the noun phrase $n p_{1}$ is represented as a vector $v$ using the following formula, where the suffix "_PP" is added to each $n$ if $n p_{1}$ is a bridging anaphor and its semantic type is not time:

$$
v=\frac{\sum_{n \in N} \text { embeddings_bridging } g_{n}}{|N|}
$$

The underlying intuition of adding NP modifications to the list $N$ is that the above mentioned modifiers also represent core semantics of an NP, therefore we should consider them when selecting antecedents for bridging anaphors. For instance, as shown in Table 4, for Example 1, the cosine similarity between $\{$ arrangements $\mathbf{P P}\}$ and 
$\{$ products $\}$ is 0.06 , while the cosine similarity between $\{$ distribution_PP, arrangements_PP $\}$ and \{dialysis, products\} is 0.17 .

If none of the words in $N$ is present in embeddings_bridging, we simply neglect the noun phrase $n p 1$. Note that we do not add the suffix "_PP" to a bridging anaphor representing time information, because such an anaphor is likely to have the same semantic type antecedent (see Example 2). Therefore we use semantic similarity instead of relatedness to find its antecedent.

\section{Experiments}

\subsection{Dataset}

For the task of bridging anaphora resolution, we use the dataset ISNotes ${ }^{5}$ released by Markert et al. (2012). This dataset contains around 11,000 NPs annotated for information status including 663 bridging NPs and their antecedents in 50 texts taken from the WSJ portion of the OntoNotes corpus (Weischedel et al., 2011). As stated in Section 2, bridging anaphors in ISNotes are not limited to definite NPs as in previous work (Poesio et al., 1997, 2004; Lassalle and Denis, 2011). The semantic relations between anaphor and antecedent in the corpus are quite diverse: only $14 \%$ of anaphors have a part-of/attribute-of relation with the antecedent and only $7 \%$ of anaphors stand in a set relationship to the antecedent. $79 \%$ of anaphors have "other" relation with their antecedents. This includes encyclopedic or frame relations such as restaurant - the waiter as well as context-specific relations such as palms - the thieves. In Example 1, "dialysis products" is the "theme" of the distribution arrangements. More specifically, "dialysis products" belongs to the frame element "Individuals" in the "Dispersal" frame that is triggered by "distribution arrangements".

\subsection{Experimental Setup}

Following Hou et al. (2013b)'s experimental setup, we resolve bridging anaphors to entity antecedents. Entity information is based on the OntoNotes coreference annotation. We also use the OntoNotes named entity annotation to assign NPs the semantic type "time" if their entity types are "date" or "time".

\footnotetext{
${ }^{5}$ http://www.h-its.org/en/research/nlp/ isnotes-corpus
}

In Hou et al. (2013b), features are extracted by using entity information. For instance, the raw hit counts of the preposition pattern query (e.g., arrangements of products) for a bridging anaphor $a$ and its antecedent candidate $e$ is the maximum count among all instantiations of $e$. In our experiments, we simply extend the list of antecedent candidates $E_{a}$ (described in Section 4) to include all instantiations of the original entities in $E_{a}$. Note that our simple antecedent candidate selection strategy (described in Section 4) allows us to include $76 \%$ of NP antecedents compared to $77 \%$ in pairwise model III from Hou et al. (2013b) where they add top $10 \%$ salient entities as additional antecedent candidates. In Hou et al. (2013b), salient entities on each text are measured through the lengths of the coreference chains based on the gold coreference annotation.

Following Hou et al. (2013b), we measure accuracy on the number of bridging anaphors, instead of on all links between bridging anaphors and their antecedent instantiations. We calculate how many bridging anaphors are correctly resolved among all bridging anaphors.

\subsection{Using NP Head Alone}

Given an anaphor $a$ and its antecedent candidate list $E_{a}$, we predict the most related NP among all NPs in $E_{a}$ as the antecedent for $a^{6}$. The relatedness is measured via cosine similarity between the head of the anaphor (plus the postfix "_PP" if the anaphor is not a time expression) and the head of the candidate. We run experiments on the following four word embeddings: the original GloVe vectors trained on Gigaword and Wikipedia 2014 dump (GloVe_GigaWikil4), GloVe vectors that we trained on Gigaword only (GloVe_Giga), word vectors from Hou (2018) (embeddings_PP), and our word representation resource described in Section 3.2 (embeddings_bridging). Note that for the first two word vectors, we do not add the suffix "PP" to the anaphor's head since such words do not exist in GloVe_GigaWikil4 and GloVe_Giga.

Table 5 lists the results for bridging anaphora resolution based on different word representation resources ${ }^{7}$. We notice that there is not

\footnotetext{
${ }^{6}$ In case of a tie, the closest one is chosen to be the predicted antecedent.

${ }^{7}$ Note that the results for the first three word embeddings are slight better than the ones reported in Hou (2018). This is due to the improved antecedent candidate selection strategy described in Section 4.
} 


\begin{tabular}{l|l}
\hline & acc \\
\hline GloVe_GigaWikil4 & 21.42 \\
GloVe_Giga & 21.87 \\
embeddings_PP & $\mathbf{3 3 . 0 3}$ \\
embeddings_bridging & $\mathbf{3 4 . 8 4}$
\end{tabular}

Table 5: Results of using NP head alone for bridging anaphora resolution based on different word representation resources. Bold indicates statistically significant differences over the baselines (two-sided paired approximate randomization test, $p<0.01$ ).

much difference between GloVe_GigaWikil4 and GloVe_Giga. We find that using embeddings_PP achieves an accuracy of $33.03 \%$ on the ISNotes corpus, which outperforms the results based on GloVe_GigaWikil4 and GloVe_Giga by a large margin. Using embeddings_bridging further improves the result by $1.8 \%$. Although the improvement is not significant, we suspect that the representations for words without the suffix ".PP" in embeddings_bridging are more accurate because they are trained on many more instances in the vanilla GloVe vectors (GloVe_GigaWiki14).

\subsection{Using NP Head + Modifiers}

We carried out experiments using the deterministic algorithm described in Section 4 together with different word embeddings. Again we do not add the suffix "PP" to the bridging anaphors for GloVe_GigaWikil4 and GloVe_Giga.

Table 6 lists the best results of the two models for bridging anaphora resolution from Hou et al. (2013b). pairwise model III is a pairwise mentionentity model based on various semantic, syntactic and lexical features. MLN model II is a joint inference framework based on Markov logic networks (Domingos and Lowd, 2009). It models that semantically or syntactically related anaphors are likely to share the same antecedent and achieves an accuracy of $41.32 \%$ on the ISNotes corpus.

The results for GloVe_GigaWikil4 and GloVe_Giga are similar on two settings (using NP head vs. using NP head + modifiers). For embeddings_PP, the result on using NP head + modifiers $(31.67 \%)$ is worse than the result on using NP head (33.03\%). However, if we apply embeddings $P P$ to a bridging anaphor's head and modifiers, and only apply embeddings_PP to the head noun of an antecedent candidate, we get an accuracy of $34.53 \%$. Although the differences are not significant, it confirms that the information

\begin{tabular}{l|l}
\hline & acc \\
\hline models from Hou et al. (2013b) \\
\hline pairwise model III & 36.35 \\
MLN model II & $\mathbf{4 1 . 3 2}$ \\
\hline \multicolumn{2}{c}{ NP head + modifiers } \\
\hline GloVe_GigaWikil4 & 20.52 \\
GloVe_Giga & 20.81 \\
embeddings_PP & 31.67 \\
embeddings_bridging & $\mathbf{3 9 . 5 2}$ \\
\hline
\end{tabular}

Table 6: Results of using NP head plus modifications in different word representations for bridging anaphora resolution compared to the best results of two models from Hou et al. (2013b). Bold indicates statistically significant differences over the other models (two-sided paired approximate randomization test, $p<0.01$ )

from the modifiers of the antecedent candidates in embeddings $P P$ hurts the performance. This corresponds to our observations in the previous section that the representations for words without the suffix ".PP" in embeddings_PP are not as good as in embeddings_bridging due to less training instances.

Finally, our method based on embeddings_bridging achieves an accuracy of $39.52 \%$, which is competitive to the best result (41.32\%) reported in Hou et al. (2013b). There is no significant difference between NP head + modifiers based on embeddings_bridging and MLN model II (randomization test with $p<0.01$ ).

To gain an insight into the contribution of embeddings_bridging on different relation types, we analyze the results of our method using embeddings_bridging on three relation types: set-of, part-of, and other. The accuracies on these three relation types are $17.78 \%, 50.0 \%$, and $39.16 \%$, respectively. This suggests that in the future we should include more context for bridging anaphors that hold the set-of relation to their antecedents, because the head nouns of such anaphors often do not bear any specific meanings (e.g., Another).

\subsection{Analysis of Modifiers}

To better understand the role of NP modifiers in our method, we carried out experiments on $\mathrm{em}$ beddings_bridging using different set of modifiers (see Table 7). It seems that among all three types of modifiers, compared to using NP head alone, adding noun modifiers has the positive impact $36.65 \%$ on NP head + noun modifiers vs. $34.84 \%$ on $N P$ head). Although adding only ad- 


\begin{tabular}{l|l}
\hline embeddings_bridging & acc \\
\hline NP head & 34.84 \\
+ all modifiers & 39.52 \\
\hline + noun modifiers & $\mathbf{3 6 . 6 5}$ \\
+ adjective modifiers & 34.84 \\
+ ed/ing participle modifiers & 34.84 \\
+ noun\&adjective modifiers & $\mathbf{3 8 . 3 1}$ \\
+ noun\&ed/ing participle modifiers & $\mathbf{3 6 . 8 0}$ \\
+ adjective\&ed/ing participle modifiers & 34.84 \\
\hline
\end{tabular}

Table 7: Results of using NP head plus different modifications in embeddings_bridging.

jective modifiers does not have influence on results, combining them with noun modifiers yields some improvement over adding only noun modifiers $(38.31 \%$ on NP head + noun\&adjective modifiers vs. $36.65 \%$ on NP head + noun modifiers). On the other hand, ed/ing participle modifiers only have a small positive impact over NP head + noun modifiers when combining with noun modifiers.

\subsection{Combining NP Head + Modifiers with MLN II}

For bridging anaphora resolution, Hou (2018) integrates a much simpler deterministic approach by combining an NP head with its noun modifiers (appearing before the head) based on $\mathrm{em}$ beddings_PP into the MLN II system (Hou et al., 2013b). Similarly, we add a constraint on top of MLN II using our deterministic approach (NP head + modifiers) based on embeddings_bridging. Table 8 lists the results of different systems ${ }^{8}$ for bridging anaphora resolution in ISNotes. It shows that combining our deterministic approach (NP Head + modifiers) with MLN II slightly improves the result compared to Hou (2018).

Although combining NP Head + modifiers with $M L N I I$ achieves significant improvement over NP Head + modifiers, we think the latter has its own value. Our deterministic algorithm is simpler and more efficient compared to MLN model II + em-

\footnotetext{
${ }^{8} \mathrm{We}$ also reimplement the algorithms from Schulte im Walde (1998) and Poesio et al. (2004) as baselines (Table 8). Schulte im Walde (1998) resolved bridging anaphors to the closest antecedent candidate in a high-dimensional space. We use the 2,000 most frequent words (adjectives, common nouns, proper nouns, and lexical verbs) from Gigaword as the context words. Poesio et al. (2004) applied a pairwise model combining lexical semantic features and salience features to perform mereological bridging resolution in the GNOME corpus. We use a Naive Bayes classifier with standard settings in WEKA (Witten and Frank, 2005) and apply the best first strategy to select the antecedent for each anaphor.
}

beddings_bridging, which contains many complicated features and might be hard to migrate to other bridging corpora. Moreover, our algorithm is "unsupervised" and requires no training when applied to other English bridging corpora.

\subsection{Resolving Bridging Anaphors in Other Corpora}

Recently, two new corpora containing bridging annotation have become available. The BASHI corpus (Rösiger, 2018a) contains 459 bridging NPs and their antecedents in 50 World Street Journal articles. Similar to ISNotes, BASHI includes both definite and indefinite referential bridging anaphors. In addition, comparative anaphora is also considered as bridging anaphora in BASHI.

Another new corpus for bridging is the second release of the ARRAU corpus, which contains 5,512 bridging pairs in three different domains (Poesio et al., 2018). However, most bridging links in ARRAU are purely lexical bridging pairs, and only a small subset of the annotated pairs contains truly anaphoric bridging anaphors (Rösiger et al., 2018). Following Rösiger et al. (2018), we focus on resolving bridging anaphors in the news text domain (RST).

Based on embeddings_bridging, we apply our deterministic algorithm with small adaptations to resolve bridging anaphors to entity antecedents on the BASHI and ARRAU (RST) corpora. Specifically, for the BASHI corpus, we do not add NPs from the first sentence to the list of antecedent candidates $E_{a}$. This is because the phenomenon of globally salient antecedents being linked to all anaphors in a text is less obvious in BASHI. In addition, comparative anaphors often have the same semantic class as their antecedents, therefore we do not add the suffix "PP" to a bridging anaphor if it is a comparative anaphor.

For the ARRAU corpus, we construct the list of antecedent candidates $E_{a}$ using NPs preceding $a$ from the same sentence as well as from the previous ten sentences. Since most bridging pairs in ARRAU are lexical bridging (e.g., Tokyo - Japan, other nations - Britain) and anaphors often have the same semantic type as their antecedents, we do not add the suffix "PP" to bridging anaphors.

Table 9 lists the results of bridging anaphora resolution in the BASHI and ARRAU corpora, respectively. On the test set of the ARRAU (RST) corpus, Rösiger (2018b) proposed a modi- 


\begin{tabular}{l|l|l}
\hline & System & acc \\
\hline Baselines & Schulte im Walde (1998) & 13.68 \\
& Poesio et al. (2004) & 18.85 \\
\hline Models from & pairwise model III & 36.35 \\
Hou et al. (2013b) & MLN model II & 41.32 \\
\hline Hou (2018) & MLN model II + embeddings_PP (NP head + noun pre-modifiers) & $\mathbf{4 5 . 8 5}$ \\
\hline This work & embeddings_bridging (NP head + modifiers) & 39.52 \\
& MLN model II + embeddings_bridging (NP head + modifiers) & $\mathbf{4 6 . 4 6}$ \\
\hline
\end{tabular}

Table 8: Results of different systems for bridging anaphora resolution in ISNotes. Bold indicates statistically significant differences over the other models (two-sided paired approximate randomization test, $p<0.01$ ).

\begin{tabular}{l|l|c|l}
\hline Corpus & Bridging Type & \# of Anaphors & acc \\
\hline BASHI & referential, including comparative anaphora & 452 & 27.43 \\
BASHI & referential, excluding comparative anaphora & 344 & 29.94 \\
ARRAU (RST Train) & mostly lexical, some referential & 2,325 & 31.44 \\
ARRAU (RST Test) & mostly lexical, some referential & 639 & 32.39 \\
\hline
\end{tabular}

Table 9: Results of resolving bridging anaphors in other corpora. Number of bridging anaphors is reported after filtering out a few problematic cases on each corpus.

fied rule-based system based on Hou et al. (2014)'s work and reported an accuracy of $39.8 \%$ for bridging anaphora resolution. And our algorithm achieves an accuracy of $32.39 \%$ using only $\mathrm{em}$ beddings_bridging. Overall, the reasonable performance on these two corpora demonstrates that embeddings_bridging is a general word representation resource for bridging.

\section{Conclusions}

We improve the word representation resource $\mathrm{em}$ beddings_PP (Hou, 2018) by combining it with GloVe. The resulting word embeddings (embeddings_bridging) are a more general word representation resource for bridging. Based on embeddings_bridging, we propose a deterministic approach for choosing antecedents for bridging anaphors. We show that this simple and efficient method achieves the competitive result on bridging anaphora resolution compared to the advanced machine learning-based approach in $\mathrm{Hou}$ et al. (2013b) which is heavily dependent on a lot of carefully designed complex features. We also demonstrate that using embeddings_bridging yields better results than using embeddings_PP for bridging anaphora resolution.

For the task of bridging anaphora resolution, Hou et al. (2013b) pointed out that considering only head noun knowledge is not enough and future work needs to explore wider context to re- solve context-specific bridging relations. In this work we explore the context within NPs-that is, we combine the semantics of certain modifications and the head by vector average using $\mathrm{em}$ beddings_bridging. But in some cases, knowledge about NPs themselves is not enough for resolving bridging. For instance, in Example 3, knowing that any loosening has the ability to "rekindle inflation" from the context of the second sentence can help us to find its antecedent "the high rates" (which is used to against inflation).

(3) Chancellor of the Exchequer Nigel Lawson views the high rates as his chief weapon against inflation, which was ignited by tax cuts and loose credit policies in 1986 and 1987. Officials fear that any loosening this year could rekindle inflation or further weaken the pound against other major currencies.

In the future, we will study how to integrate context outside of NPs for the task of choosing antencedents for bridging anaphors. Also we hope that our word representation resource will facilitate other related research problems such as semantic role labeling.

\section{Acknowledgments}

The author appreciates the valuable feedback from the anonymous reviewers and would like to thank Massimo Poesio for sharing the ARRAU corpus. 


\section{References}

I.I. Bejar, R. Chaffin, and S.E. Embretson. 1991. Cognitive and psychometric analysis of analogical problem solving. Springer-Verlag.

Danqi Chen, Jason Bolton, and Christopher D. Manning. 2016. A thorough examination of the CNN/Daily mail reading comprehension task. In Proceedings of the 54th Annual Meeting of the Association for Computational Linguistics, Berlin, Germany, 7-12 August 2016, pages 2358-2367.

Pedro Domingos and Daniel Lowd. 2009. Markov Logic: An Interface Layer for Artificial Intelligence. Morgan Claypool Publishers.

Yufang Hou. 2016. Incremental fine-grained information status classification using attention-based LSTMs. In Proceedings of the 26th International Conference on Computational Linguistics, Osaka, Japan, 11-16 December 2016, pages 1880-1890.

Yufang Hou. 2018. Enhanced word representations for bridging anaphora resolution. In Proceedings of the 2018 Conference of the North American Chapter of the Association for Computational Linguistics: Human Language Technologies, New Orleans, Louisiana, 1-6 June 2018, pages 1-7.

Yufang Hou, Katja Markert, and Michael Strube. 2013a. Cascading collective classification for bridging anaphora recognition using a rich linguistic feature set. In Proceedings of the 2013 Conference on Empirical Methods in Natural Language Processing, Seattle, Wash., 18-21 October 2013, pages 814820 .

Yufang Hou, Katja Markert, and Michael Strube. 2013b. Global inference for bridging anaphora resolution. In Proceedings of the 2013 Conference of the North American Chapter of the Association for Computational Linguistics: Human Language Technologies, Atlanta, Georgia, 9-14 June 2013, pages 907-917.

Yufang Hou, Katja Markert, and Michael Strube. 2014. A rule-based system for unrestricted bridging resolution: Recognizing bridging anaphora and finding links to antecedents. In Proceedings of the 2014 Conference on Empirical Methods in Natural Language Processing, Doha, Qatar, 25-29 October 2014, pages 2082-2093.

David A. Jurgens, Peter D. Turney, Saif M. Mohammad, and Keith J. Holyoak. 2012. Semeval-2012 task 2: Measuring degrees of relational similarity. In Proceedings of the First Joint Conference on Lexical and Computational Semantics, Montréal, Canada, 7-8 June 1999, pages 356-364.

Emmanuel Lassalle and Pascal Denis. 2011. Leveraging different meronym discovery methods for bridging resolution in French. In Proceedings of the 8th Discourse Anaphora and Anaphor Resolution Colloquium (DAARC 2011), Faro, Algarve, Portugal, 6 7 October 2011, pages 35-46.
Kenton Lee, Luheng He, Mike Lewis, and Luke Zettlemoyer. 2017. End-to-end neural coreference resolution. In Proceedings of the 2017 Conference on Empirical Methods in Natural Language Processing, Copenhagen, Denmark, 7-11 November 2017, pages 188-197.

Katja Markert, Yufang Hou, and Michael Strube. 2012. Collective classification for fine-grained information status. In Proceedings of the 50th Annual Meeting of the Association for Computational Linguistics, Jeju Island, Korea, 8-14 July 2012, pages 795-804.

Katja Markert, Malvina Nissim, and Natalia N. Modjeska. 2003. Using the web for nominal anaphora resolution. In Proceedings of the EACL Workshop on the Computational Treatment of Anaphora. Budapest, Hungary, 14 April 2003, pages 39-46.

Tomas Mikolov, Ilya Sutskever, Kai Chen, Gregory S. Corrado, and Jeffrey Dean. 2013. Distributed representations of words and phrases and their compositionality. In Advances in Neural Information Processing Systems 26 (NIPS 2013), pages 3111-3119.

Courtney Napoles, Matthew Gormley, and Benjamin Van Durme. 2012. Annotated Gigaword. In Proceedings of the Joint Workshop on Automatic Knowledge Base Construction \& Web-scale Knowledge Extraction ( $A K B C$-WEKEX) Montréal, Québec, Canada, 7-8 June 2012, pages 95-100.

Robert Parker, David Graff, Junbo Kong, Ke Chen, and Kazuaki Maeda. 2011. English Gigaword Fifth Edition. LDC2011T07.

Jeffrey Pennington, Richard Socher, and Christopher D. Manning. 2014. Glove: Global vectors for word representation. In Proceedings of the 2014 Conference on Empirical Methods in Natural Language Processing, Doha, Qatar, 25-29 October 2014, pages 1532-1543.

Massimo Poesio, Yulia Grishina, Varada Kolhatkar, Nafise Sadat Moosavi, Ina Rösiger, Adam Roussel, Fabian Simonjetz, Alexandra Uma, Olga Uryupina, Juntao Yu, and Heike Zinsmeister. 2018. Anaphora resolution with the ARRAU corpus. In Proceedings of the Workshop on Computational Models of Reference, Anaphora and Coreference. New Orleans, Louisiana, June 6, 2018, pages 11-22.

Massimo Poesio, Rahul Mehta, Axel Maroudas, and Janet Hitzeman. 2004. Learning to resolve bridging references. In Proceedings of the 42nd Annual Meeting of the Association for Computational Linguistics, Barcelona, Spain, 21-26 July 2004, pages 143-150.

Massimo Poesio and Renata Vieira. 1998. A corpusbased investigation of definite description use. Computational Linguistics, 24(2):183-216.

Massimo Poesio, Renata Vieira, and Simone Teufel. 1997. Resolving bridging references in unrestricted 
text. In Proceedings of the ACL Workshop on Operational Factors in Practical, Robust Anaphora Resolution for Unrestricted Text, Madrid, Spain, July 1997, pages 1-6.

Tim Rocktäschel, Edward Grefenstette, Karl Moritz Hermann, Tomas Kocisky, and Phil Blunsom. 2016. Reasoning about entailment with neural attention. In Proceedings of the 4th International Conference on Learning Representations, San Juan, Puerto Rico, 2-4 May 2016.

Ina Rösiger. 2018a. BASHI: A corpus of wall street journal articles annotated with bridging links. In Proceedings of the 11th International Conference on Language Resources and Evaluation, Miyazaki, Japan, 7-12 May 2018, pages 382-388.

Ina Rösiger. 2018b. Rule- and learning-based methods for bridging resolution in the ARRAU corpus. In Proceedings of the Workshop on Computational Models of Reference, Anaphora and Coreference. New Orleans, Louisiana, June 6, 2018, pages 23-33.

Ina Rösiger, Arndt Riester, and Jonas Kuhn. 2018. Bridging resolution: Task definition, corpus resources and rule-based experiments. In Proceedings of the 27th International Conference on Computational Linguistics, Santa Fe, New-Mexico, USA, 20-26 August 2018, pages 3516-3528.

Sabine Schulte im Walde. 1998. Resolving bridging descriptions in high-dimensional space. Master's thesis, University of Edinburgh, Centre for Cognitive Science.

Ralph Weischedel, Martha Palmer, Mitchell Marcus, Eduard Hovy, Sameer Pradhan, Lance Ramshaw, Nianwen Xue, Ann Taylor, Jeff Kaufman, Michelle Franchini, Mohammed El-Bachouti, Robert Belvin, and Ann Houston. 2011. OntoNotes release 4.0. LDC2011T03, Philadelphia, Penn.: Linguistic Data Consortium.

Ian H. Witten and Eibe Frank. 2005. Data Mining: Practical Machine Learning Tools and Techniques, 2nd edition. Morgan Kaufmann, San Francisco, Cal.

Alisa Zhila, Scott Wen-tau Yih, Geoffrey Zweig, Chris Meek, and Tomas Mikolov. 2013. Combining heterogeneous models for measuring relational similarity. In Proceedings of the 2013 Conference of the North American Chapter of the Association for Computational Linguistics: Human Language Technologies, Atlanta, Georgia, 9-14 June 2013, pages 1000-1009. 